\title{
An Evaluation for the Probability Density of the First Hitting Time
}

\author{
Shih-Yu Shen ${ }^{1 *}$, Yi-Long Hsiao ${ }^{2}$ \\ ${ }^{1}$ Department of Mathematics, National Cheng-Kung University, Tainan, Chinese Taipei \\ ${ }^{2}$ Department of Finance, National Dong Hwa University, Hualien County, Chinese Taipei \\ Email: *shen@mail.ncku.edu.tw
}

Received March 7, 2013; revised April 7, 2013; accepted April 14, 2013

Copyright (C) 2013 Shih-Yu Shen, Yi-Long Hsiao. This is an open access article distributed under the Creative Commons Attribution License, which permits unrestricted use, distribution, and reproduction in any medium, provided the original work is properly cited.

\section{ABSTRACT}

Let $h(t)$ be a smooth function, $B_{t}$ a standard Brownian motion and $\tau_{h}=\inf \left\{\tau ; B_{\tau}=h(\tau)\right\}$ the first hitting time. In this paper, new formulations are derived to evaluate the probability density of the first hitting time. If $u(x, t)$ denotes the density function of $x=B_{t}$ for $t<\tau_{h}$, then $u_{x x}=2 u_{t}$ and $u(h(t), t)=0$. Moreover, the hitting time density $d_{h}(t)$ is $\frac{1}{2} u_{x}(h(t), t)$. Applying some partial differential equation techniques, we derive a simple integral equation for $d_{h}(t)$. Two examples are demonstrated in this article.

Keywords: Brownian Motion; First Hitting Time; Heat Equation; Boundary Value Problem

\section{Introduction}

Since the publication of Black and Scholes' [1], and Merton's [2] papers in 1973, a stock price following a geometric Brownian motion becomes the standard model for the dynamics of a stock price. Therefore, the calculations for the first hitting time get important in the field of finance recently [3].

Let $h(t)$ be a smooth function for $t \geq 0$, and $B=$ $\left\{B_{t} ; t \geq 0\right\}$ a standard Brownian motion. The first hitting time $\tau_{h}$ is defined as $\inf \left\{t ; B_{t}=h(t) \mid B_{0}=0\right\}$. It is known that $\tau_{h}$ has a continuous density [4]. Sometime we call the function $h(t)$ or the curve $x=h(t)$ the barrier. For a constant barrier, the result has been wellknown for a long time. In this case, the density is

$$
\frac{|h|}{\sqrt{2 \pi t^{3}}} \exp \left(-\frac{h^{2}}{2 t}\right) \text { ([5], Chapter 9). The distribution of the }
$$
hitting time for non-constant barrier was considered by many authors; for example in [6-9]. In [6], Cuzick developed an asymptotic estimate for the first hitting density with a general barrier. In $[7,8]$, the authors' formulas contain an expected value of a Brownian function. The formulations in [7] are hard to see how the density for a

"Corresponding author. general barrier is evaluated. Although the expected value in [8] can be evaluated by solving a partial differential equation, using a numerical method to compute the value is still not easy. In [9], the density function for parabolic barriers was expressed analytically in terms of Airy functions. In this article, we derive new exact formulations for the hitting density with a general barrier. Thus, partial differential equation (PDE) techniques may be applied to evaluate the density function of the first hitting time. Let $u(x, t)$ be the probability density of $\left\{B_{t}=x, t<\tau_{h} \mid B_{0}=0\right\}$; i.e. $u(x, t) \mathrm{d} x=\boldsymbol{P}\left\{B_{t} \in(x, x+\mathrm{d} x), t<\tau_{h} \mid B_{0}=0\right\}$. It will be shown that $u(x, t)$ is the solution of an initialboundary value problem of a heat equation, and the hitting density is $\frac{-h(0)}{2|h(0)|} \frac{\partial}{\partial x} u(h(t), t)$. Our derivation results a simple integral equation for the density function.

In Section 2, we show that the density function of the first hitting time can be evaluated though solving an initial-boundary value problem of the heat equation. Then, the density function will be the solution of a simple integral equation. In Section 3, a couple of examples are solved by PDE techniques to demonstrate the justification of the new method. The last section is the conclusion. 


\section{The Boundary Value Problem}

Let $B_{t}, B_{0}=0$, a standard Brownian motion, $h(t)$ be a smooth function and $\tau_{h}=\inf \left\{t ; B_{t}=h(t)\right\}$ the first hitting time. We consider $h(0)>0$ first. Let $p(x, t)$ denote the probability $\boldsymbol{P}\left\{B_{t}<x, t<\tau_{h}\right\}$, and $u(x, t)$ denote the density function $\frac{\partial}{\partial t} p(x, t)$. Surely, $u(x, t)$ fulfills the heat equation, $u_{x x}=2 u_{t}$ ([10], p. 352). Nevertheless, in this section, it will be proven with another way. To derive the formulations for $u(x, t)$, we consider the hitting problem at discrete times $\left\{t_{1}, t_{2}, \cdots, t_{n}\right\}$, where $t_{i}=i \frac{t}{n}$. Let $p_{i}(x)$ denote

$$
\boldsymbol{P}\left\{B_{t_{i}}<x,\left(B_{t_{j}}<h\left(t_{j}\right) ; j=1,2, \cdots, i\right)\right\},
$$

and $u_{i}(x)$ denote $\frac{\mathrm{d}}{\mathrm{d} x} p_{i}(x)$. Therefore,

$$
u_{i+1}(x)=\int_{-\infty}^{h\left(t_{i}\right)} G(x, \xi) u_{i}(\xi) \mathrm{d} \xi
$$

where $G(x, \xi)=1 \sqrt{2 \pi \Delta t} \mathrm{e}^{-\frac{(x-\xi)^{2}}{2 \Delta t}}$ and $\Delta t=t / n$. Taking the limit, we have

$$
\lim _{n \rightarrow \infty} p_{n}(x)=p(x, t)
$$

and

$$
\lim _{n \rightarrow \infty} u_{n}(x)=u(x, t)
$$

We will show that $u(x, t)$ satisfies the heat equation. Integrating Equation (1), we have

$$
p_{i+1}(x)=\int_{-\infty}^{x} \int_{-\infty}^{h(i)} \frac{1}{\sqrt{2 \pi \Delta t}} \mathrm{e}^{-\frac{(\bar{x}-\xi)^{2}}{2 \Delta t}} u_{i}(\xi) \mathrm{d} \xi \mathrm{d} \bar{x},
$$

for $x<h\left(t_{i+1}\right)$. The probability difference between two steps is

$$
\begin{aligned}
& p_{i+1}(x)-p_{i}(x) \\
& =\int_{-\infty}^{x} \int_{-\infty}^{h\left(t_{i}\right)} \frac{1}{\sqrt{2 \pi \Delta t}} \mathrm{e}^{-\frac{(\bar{x}-\xi)^{2}}{2 \Delta t}} u_{i}(\xi) \mathrm{d} \xi \mathrm{d} \bar{x} \\
& -\int_{-\infty}^{x} u_{i}(\bar{x}) \int_{-\infty}^{\infty} \frac{1}{\sqrt{2 \pi \Delta t}} \mathrm{e}^{-\frac{(\bar{x}-\xi)^{2}}{2 \Delta t}} \mathrm{~d} \xi \mathrm{d} \bar{x} \\
& =\int_{-\infty}^{x} \int_{-\infty}^{h\left(t_{i}\right)} \frac{1}{\sqrt{2 \pi \Delta t}} \mathrm{e}^{-\frac{(\bar{x}-\xi)^{2}}{2 \Delta t}}\left(u_{i}(\xi)-u_{i}(\bar{x})\right) \mathrm{d} \xi \mathrm{d} \bar{x} \\
& -\int_{-\infty}^{x} \int_{h\left(t_{i}\right)}^{\infty} \frac{1}{\sqrt{2 \pi \Delta t}} \mathrm{e}^{-\frac{(\bar{x}-\xi)^{2}}{2 \Delta t}} u_{i}(\bar{x}) \mathrm{d} \xi \mathrm{d} \bar{x} .
\end{aligned}
$$

Using a substitution, $v=\bar{x}-\xi$, we have

$$
\begin{aligned}
& p_{i+1}(x)-p_{i}(x) \\
& =\int_{-\infty}^{\infty} \int_{-\infty}^{a(v)}-\frac{1}{\sqrt{2 \pi \Delta t}} \mathrm{e}^{-\frac{v^{2}}{2 \Delta t}}\left(u_{i}(\bar{x})-u_{i}(\bar{x}-v)\right) \mathrm{d} \bar{x} \mathrm{~d} v \\
& -\int_{-\infty}^{\infty} \int_{a(v)}^{x} \frac{1}{\sqrt{2 \pi \Delta t}} \mathrm{e}^{-\frac{v^{2}}{2 \Delta t}} u_{i}(\bar{x}) \mathrm{d} \bar{x} \mathrm{~d} v
\end{aligned}
$$

where

$$
a(v)= \begin{cases}x, & \text { if } v \geq x-h\left(t_{i}\right) \\ v+h\left(t_{i}\right), & \text { if } v<x-h\left(t_{i}\right) .\end{cases}
$$

Consequently,

$$
\begin{aligned}
& p_{i+1}(x)-p_{i}(x) \Delta t \\
& =\int_{-\infty}^{\infty} \frac{-v}{\Delta t \sqrt{2 \pi \Delta t}} \mathrm{e}^{-\frac{v^{2}}{2 \Delta t}} \int_{-\infty}^{a(v)} \frac{u_{i}(\bar{x})-u_{i}(\bar{x}-v)}{v} \mathrm{~d} \bar{x} \mathrm{~d} v \\
& -\int_{-\infty}^{\infty} \frac{v}{\Delta t \sqrt{2 \pi \Delta t}} \mathrm{e}^{-\frac{v^{2}}{2 \Delta t}} \frac{1}{v} \int_{a(v)}^{x} u_{i}(\bar{x}) \mathrm{d} \bar{x} \mathrm{~d} v \\
& =\int_{-\infty}^{\infty} \frac{-v}{\Delta t \sqrt{2 \pi \Delta t}} \mathrm{e}^{-\frac{v^{2}}{2 \Delta t}} \frac{1}{v} \int_{a(v)-v}^{a(v)} u_{i}(\bar{x}) \mathrm{d} \bar{x} \mathrm{~d} v \\
& -\int_{-\infty}^{\infty} \frac{v}{\Delta t \sqrt{2 \pi \Delta t}} \mathrm{e}^{-\frac{v^{2}}{2 \Delta t}} \frac{1}{v} \int_{a(v)}^{x} u_{i}(\bar{x}) \mathrm{d} \bar{x} \mathrm{~d} v
\end{aligned}
$$

Note that, if $g(v)$ is continuous and bounded

$$
\lim _{t \rightarrow 0^{+}} \int_{-\infty}^{\infty} \frac{1}{\sqrt{\pi t}} \mathrm{e}^{-\frac{v^{2}}{t}} g(v) \mathrm{d} v=g(0),
$$

([11], p. 9) and, therefore, if $g(v) \in \mathcal{C}^{1}$

$$
\lim _{t \rightarrow 0^{+}} \int_{-\infty}^{\infty} \frac{2 v}{t \sqrt{\pi t}} \mathrm{e}^{-\frac{v^{2}}{t}} g(v) \mathrm{d} v=g^{\prime}(0) .
$$

Let

$$
g(v)= \begin{cases}u_{i}(x), & \text { if } v=0 \\ \frac{1}{v} \int_{a(v)-v}^{a}(v) u_{i}(\bar{x}) \mathrm{d} \bar{x}, & \text { if } v \neq 0 .\end{cases}
$$

The function $g(v)$ is continuous and bounded. Moreover, $g(v)$ is differentiable, since

$$
\begin{aligned}
g^{\prime}(0) & =\lim _{v \rightarrow 0} \frac{1}{v}\left(g(v)-u_{i}(x)\right) \\
& =\lim _{v \rightarrow 0} \frac{1}{v^{2}} \int_{a(v)-v}^{a(v)} u_{i}(\bar{x})-u_{i}(x) \mathrm{d} \bar{x} \\
& =\lim _{v \rightarrow 0} \frac{1}{v^{2}} \int_{x-v}^{x} u_{i}(\bar{x})-u_{i}(x) \mathrm{d} \bar{x} \\
& =\lim _{v \rightarrow 0} \frac{1}{v^{2}}\left(-u_{i}^{\prime}(x) \frac{v^{2}}{2}+O\left(v^{3}\right)\right)=\frac{1}{2} \frac{\mathrm{d}}{\mathrm{d} x} u_{i}(x)
\end{aligned}
$$

Thus, when $\Delta t$ approaches 0 , the first term of the 
right-hand side of Equation (6) is $-\frac{1}{2} \frac{\mathrm{d}}{\mathrm{d} x} u_{i}(x)$. The second term is 0 , because $x<h\left(t_{i}\right)$ and, therefore, $a(v)=x$ when $|v|$ is small. Letting $\Delta t$ approach 0 , we have

$$
\frac{\partial}{\partial t} p(x, t)=\frac{1}{2} u_{x}(x, t)
$$

Since $\frac{\partial}{\partial t} p(x, t)=u(x, t)$, differentiating both sides of Equation (7) with respect to $x$, we have the partial differential equation

$$
u_{t}(x, t)=\frac{1}{2} u_{x x}(x, t) .
$$

The barrier $h(t)$ is assumed to be differentiable, and, therefore, there exists an positive number $\kappa$ not depending on $\Delta t$ such that $\left|h\left(t_{i+1}\right)-h\left(t_{i}\right)\right|<\kappa \Delta t$. Consider the probability density near the boundary $h\left(t_{i+1}\right)$

$$
\begin{aligned}
& u_{i+1}\left(h\left(t_{i}\right)+s \Delta t\right) \\
& =\int_{-\infty}^{h\left(t_{i}\right)} \frac{1}{\sqrt{2 \pi \Delta t}} \mathrm{e}^{-\frac{\left(h\left(t_{i}\right)+s \Delta t-\xi\right)^{2}}{2 \Delta t}} u_{i}(\xi) \mathrm{d} \xi \\
& =\mathrm{e}^{-\frac{s^{2} \Delta t}{2}} \int_{-\infty}^{h\left(t_{i}\right)} \frac{1}{\sqrt{2 \pi \Delta t}} \mathrm{e}^{-\frac{\left(h\left(t_{i}\right)-\xi\right)^{2}}{2 \Delta t}}\left(\mathrm{e}^{\frac{2 s\left(h\left(t_{i}\right)-\xi\right)}{2}} u_{i}(\xi)\right) \mathrm{d} \xi,
\end{aligned}
$$

where $|s|<\kappa$. Note that

$$
\lim _{\Delta t \rightarrow 0} \int_{h\left(t_{i}\right)}^{\infty} \frac{1}{\sqrt{2 \pi \Delta t}} \mathrm{e}^{-\frac{\left(h\left(t_{i}\right)-\tau\right)^{2}}{2 \Delta t}} f(\tau) \mathrm{d} \tau=\frac{1}{2} f\left(h\left(t_{i}\right)\right)
$$

When $s(\Delta t)=\frac{h\left(t_{i+1}\right)-h\left(t_{i}\right)}{\Delta t}$,

$$
\lim _{\Delta t \rightarrow 0} u_{i+1}\left(h\left(t_{i+1}\right)\right)=\frac{1}{2} u_{i}\left(h\left(t_{i}\right)\right) .
$$

Consequently,

$$
\lim _{\Delta t \rightarrow 0} u_{n}\left(h\left(t_{n}\right)\right)=\lim _{n \rightarrow \infty}\left(\frac{1}{2}\right)^{n} u_{0}\left(h\left(t_{0}\right)\right) .
$$

We have the boundary condition

$$
u(h(t), t)=0
$$

Therefore, we have a proposition as follows:

\section{Proposition 1}

The density function $u(x, t)$ is subject to the initialboundary value problem:

$$
\begin{gathered}
u_{x x}(x, t)=2 u_{t}(x, t), \\
u(h(t), t)=0, \\
u(x, 0)=\delta(x),
\end{gathered}
$$

where $\delta(x)$ is the Dirac delta function. The initialboundary value problem is mathematically well-post. The hitting probability $p_{h}(t)$,

$$
p_{h}(t)=1-\int_{-\infty}^{h(t)} u(x, t) \mathrm{d} x .
$$

Then, the hitting density $d_{h}(t)$

$$
\begin{aligned}
d_{h}(t) & =\frac{\mathrm{d}}{\mathrm{d} t} p_{h}(t) \\
& =-\frac{\mathrm{d}}{\mathrm{d} t} h(t) u(h(t), t)-\int_{-\infty}^{h(t)} \frac{\partial}{\partial t} u(x, t) \mathrm{d} x .
\end{aligned}
$$

Substituting Equations (8) and (10) into Equation (13), we have

$$
d_{h}(t)=-\int_{-\infty}^{h(t)} \frac{1}{2} u_{x x}(x, t) \mathrm{d} x
$$

Using integration by parts, we have

$$
d_{h}(t)=-\frac{1}{2} u_{x}(h(t), t) .
$$

Similarly, if $h(0)<0$, the hitting density will be $\frac{1}{2} u_{x}(h(t), t)$. There is an integral equation for the boundary values of a heat equation ([12], p. 219).

$$
\begin{aligned}
& \qquad \begin{aligned}
u(h(t), t)= & \int_{-\infty}^{h(0)} u(\xi, 0) G^{*}(\xi, 0, h(t), t) \mathrm{d} \xi \\
& +\int_{0}^{t} u_{x}(h(\tau), \tau) G^{*}(h(\tau), \tau, h(t), t) \\
& -u(h(\tau), \tau) G_{\xi}^{*}(h(\tau), \tau, h(t), t) \mathrm{d} \tau,
\end{aligned} \\
& \text { where } G^{*}(\xi, \tau, x, t)=\frac{1}{\sqrt{2 \pi(t-\tau)}} \mathrm{e}^{-\frac{(x-\xi)^{2}}{2(t-\tau)}} H(t-\tau) \text { and }
\end{aligned}
$$
$H(t)$ is the Heaviside step function. For problem (11), the integral equation becomes

$$
G^{*}(0,0, h(t), t)-\int_{0}^{t} 2 d_{h}(\tau) G^{*}(h(\tau), \tau, h(t), t) \mathrm{d} \tau=0,
$$

or

$$
\int_{0}^{t} \frac{2}{\sqrt{2 \pi(t-\tau)}} \mathrm{e}^{-\frac{(h(t)-h(\tau))^{2}}{2(t-\tau)}} d_{h}(\tau) \mathrm{d} \tau=\frac{1}{\sqrt{2 \pi t}} \mathrm{e}^{-\frac{x^{2}}{2 t}}
$$

Equation (16) can be solved by a numerical method easily.

\section{Examples}

Example 1: Linear boundaries.

Let $h(t)=a+b t$ with $a>0$. The initial-boundary value problem (11) has a close-form solution. The solution, which is a Green's function for the boundary $x=a+b t$, is 


$$
u(x, t)=\frac{1}{\sqrt{2 \pi t}}\left(\mathrm{e}^{-\frac{x^{2}}{2 t}}-\mathrm{e}^{-2 a b} \mathrm{e}^{-\frac{(x-2 a)^{2}}{2 t}}\right)
$$

Thus, the hitting density $d_{h}(t)$ is

$$
\begin{aligned}
& -\frac{1}{2} \frac{\partial}{\partial x} u(h(t), t) \\
& =\frac{1}{2 \sqrt{2 \pi t}}\left(\frac{a+b t}{t} \mathrm{e}^{-\frac{(a+b t)^{2}}{2 t}}+\mathrm{e}^{-2 a b} \frac{(a-b t)}{t} \mathrm{e}^{-\frac{(a-b t)^{2}}{2 t}}\right) \\
& =\frac{a}{t \sqrt{2 \pi t}} \mathrm{e}^{-\frac{(a-b t)^{2}}{2 t}}
\end{aligned}
$$

consistent with that in [8].

Example 2: First-passage time probability in an interval.

Let $\tau_{a}=\inf \left\{\tau ; \tau>t_{0}, B_{\tau}=a\right\}$ and $d_{a}(t)$ be the density function for $\tau_{a}=t$. In this example, we evaluate the probability density of the first passage time $d_{a}(t)$.

Let $\tau_{a}^{*}(\xi)=\inf \left\{\tau ; \tau>t_{0}, B_{\tau}=a \mid B_{t_{0}}=\xi\right\}$ and $d_{t}^{*}(\xi)$ be the density of $\tau_{a}^{*}=t$. Using $u^{l}(x, t ; \xi)$ and $u^{r}(x, t ; \xi)$ to denote the probability density

$\frac{\partial}{\partial x} \boldsymbol{P}\left\{B_{t}<x, t<\tau_{a}^{*} \mid B_{t_{0}}=\xi\right\}$ for $\xi<a$ and $\xi>a$ respectively, we have an initial-boundary value problem for both functions, $u^{l}$ and $u^{r}$. From the proposition, $u^{l}$ and $u^{r}$ fulfill the equations

$$
\begin{gathered}
\frac{\partial^{2}}{\partial x^{2}} u(x, t)=2 \frac{\partial}{\partial t} u(x, t) t>t_{0} \\
u(a, t)=0, t>t_{0}
\end{gathered}
$$

and

$$
u\left(x, t_{0}\right)=\delta(x-\xi)
$$

The solutions are

$$
u^{l}(x, t ; \xi)=\frac{1}{\sqrt{2 \pi\left(t-t_{0}\right)}}\left(\mathrm{e}^{-\frac{(x-\xi)^{2}}{2\left(t-t_{0}\right)}}-\mathrm{e}^{-\frac{(x-2 a+\xi)^{2}}{2\left(t-t_{0}\right)}}\right) H(a-x)
$$

and

$$
u^{r}(x, t ; \xi)=\frac{1}{\sqrt{2 \pi\left(t-t_{0}\right)}}\left(\mathrm{e}^{-\frac{(x-\xi)^{2}}{2\left(t-t_{0}\right)}}-\mathrm{e}^{-\frac{(x-2 a+\xi)^{2}}{2\left(t-t_{0}\right)}}\right) H(x-a) .
$$

The density function is

$$
d_{t}^{*}(\xi)= \begin{cases}-\frac{1}{2} \frac{\partial}{\partial x} u^{l}(a, t ; \xi), & \text { if } \xi<a \\ \frac{1}{2} \frac{\partial}{\partial x} u^{r}(a, t ; \xi), & \text { if } \xi>a\end{cases}
$$

Thus

$$
d_{t}^{*}(\xi)=\frac{|\xi-a|}{\left(t-t_{0}\right) \sqrt{2 \pi\left(t-t_{0}\right)}} \mathrm{e}^{-\frac{(\xi-a)^{2}}{2\left(t-t_{0}\right)}}
$$

The density of $\tau_{a}=t$ has to be

$$
\begin{aligned}
d_{a}(t) & =\int_{-\infty}^{\infty} d_{t}^{*}(\xi) u_{0}(\xi) \mathrm{d} \xi \\
& =\int_{-\infty}^{a} d_{t}^{*}(\xi) u_{0}(\xi) \mathrm{d} \xi+\int_{a}^{\infty} d_{t}^{*}(\xi) u_{0}(\xi) \mathrm{d} \xi,
\end{aligned}
$$

where $u_{0}(\xi)$ is the density function of $B_{t_{0}}=\xi$; i.e.

$$
u_{0}(\xi)=\frac{1}{\sqrt{2 \pi t_{0}}} \mathrm{e}^{-\frac{\xi^{2}}{2 t_{0}}}
$$

The integral in Equation (17) can be calculated.

$$
\begin{aligned}
& \int_{-\infty}^{a} d_{t}^{*}(\xi) u_{0}(\xi) \mathrm{d} \xi \\
& =\int_{-\infty}^{a} \frac{-2(\bar{x}-a)}{2 \pi \sqrt{\left(t-t_{0}\right) t_{0}}\left(t-t_{0}\right)} \mathrm{e}^{-\frac{(\bar{x}-a)^{2}}{2\left(t-t_{0}\right)}-\frac{(\bar{x})^{2}}{2 t_{0}}} \mathrm{~d} \bar{x} \\
& =\frac{-1}{\pi \sqrt{\left(t-t_{0}\right) t_{0}}\left(t-t_{0}\right)} \int_{-\infty}^{a}(\bar{x}-a) \mathrm{e}^{-\frac{(\bar{x}-\lambda a)^{2}}{2 \lambda\left(t-t_{0}\right)}-\frac{a^{2}}{2 t}} \mathrm{~d} \bar{x} \\
& =\frac{1}{\pi \sqrt{2 t \phi} t} \mathrm{e}^{-\frac{a^{2}}{2 t}}\left(\mathrm{e}^{-(\phi a)^{2}}+\sqrt{\pi \phi} a\left(1+\operatorname{erf}\left(\sqrt{\phi a^{2}}\right)\right)\right),
\end{aligned}
$$

where $\lambda=\frac{t_{0}}{t}$ and $\phi=\frac{1-\lambda}{2 t_{0}}$. Similarly,

$$
\begin{aligned}
& \int_{a}^{\infty} d_{t}^{*}(\xi) u_{0}(\xi) \mathrm{d} \xi \\
& =\frac{1}{\pi \sqrt{2 t \phi} t} \mathrm{e}^{-\frac{a^{2}}{2 t}}\left(\mathrm{e}^{-\phi a^{2}}+\sqrt{\pi \phi} a\left(1-\operatorname{erf}\left(\sqrt{\phi a^{2}}\right)\right)\right),
\end{aligned}
$$

Therefore, the hitting rate

$$
d_{a}(t)=\frac{1}{\sqrt{2 t^{3}}} \mathrm{e}^{-\frac{a^{2}}{2 t}}\left(\frac{1}{\pi \sqrt{\phi}} \mathrm{e}^{-\phi a^{2}}+\frac{a}{\sqrt{\pi}} \operatorname{erf}\left(\sqrt{\phi a^{2}}\right)\right)
$$

In the special case of $a=0$,

$$
d_{a}(t)=\frac{1}{\pi \sqrt{2 t \phi} t}=\frac{\sqrt{t_{0}}}{\pi \sqrt{t-t_{0}} t} .
$$

The probability of that the Brownian motion process takes on the value 0 at least once in the interval $\left(t_{0}, t\right)$ is

$$
\int_{t_{0}}^{t} \frac{\sqrt{t_{0}}}{\pi \tau \sqrt{\tau-t_{0}}} \mathrm{~d} \tau=\frac{2}{\pi} \arccos \left(\sqrt{\frac{t_{0}}{t}}\right) .
$$

This result is the same as in ([13], p. 191). In a simple case of $t_{0}=0$,

$$
d_{a}(t)=\frac{a}{\sqrt{2 \pi t^{3}}} \mathrm{e}^{-\frac{a^{2}}{2 t}}
$$


This is the well-known result of the density of the first hitting time for a constant barrier.

\section{Conclusions}

The proposition proposed in Section 2 may offer a simple way to evaluate the density of the hitting time with a general barrier by solving an initial-boundary value problem of the heat equation. The density function $u(x, t)$ locally satisfies the heat equation is well-known [10]. The main contribution of this paper is the derivation of the boundary condition (10). This result makes progress in the evaluation of hitting time density. Two examples with exact solutions are demonstrated in Section 3. Even though the examples may be solved by other method, the new formulations in this paper can be applied to evaluate the hitting time distribution with any smooth barrier numerically by using the integral Equation (16).

A similar result for two-dimensional problems may be expected. Let $B_{t}^{1}$ and $B_{t}^{2}$ be two standard Brownian motion, $h(x, t)=y$ a smooth surface and

$$
\tau_{h}=\inf \left\{t ; h\left(B_{t}^{1}, t\right)=B_{t}^{2}\right\}
$$

the first hitting time. If $u(x, y, t)$ presents the probability density of $\left(B_{t}^{1}, B_{t}^{2}\right)=(x, y)$ for $t<\tau_{h}$, the two-dimensional formulations may be as follows,

$$
\begin{gathered}
u_{x x}+u_{y y}=2 u_{t} \\
u(x, h(x, t), t)=0, \\
u(x, y, 0)=\delta\left(x-B_{0}^{1}\right) \delta\left(y-B_{0}^{2}\right) .
\end{gathered}
$$

As long as Equation (18) is established, the probability density of the first hitting time for two-dimensional Brownian motion may be evaluated by an analytical or numerical method.

\section{REFERENCES}

[1] F. Black and M. Scholes, "The Pricing of Options and
Corporate Liabilities," Journal of Political Economy, Vol. 81, No. 3, 1973, pp. 637-659. doi:10.1086/260062

[2] R. C. Merton, "Theory of Rational Option Pricing," Bell Journal of Economics and Management Science, Vol. 4, No. 1, 1973, pp. 141-183. doi:10.2307/3003143

[3] C. Profeta, B. Roynette and M. Yor, "Option Prices as Probabilities," Springer, New York, 2010. doi:10.1007/978-3-642-10395-7

[4] B. Ferebee, "The Tangent Approximation to One-Sided Brownian Exit Densities," Z. Wahrscheinlichkeitsth, Vol. 61, No. 3, 1982, pp. 309-326. doi:10.1007/BF00539832

[5] G. R. Grimmett and D. R. Stirzaker, "Probability and Random Processes," Oxford University Press, New York, 1982.

[6] J. Cuzick, "Boundary Crossing Probabilities for Stationary Gaussian Processes and Brownian Motion," Transactions of the American Mathematical Society, Vol. 263, No. 2, 1981, pp. 469-492. doi:10.1090/S0002-9947-1981-0594420-5

[7] J. Durbin, "The First-Passage Density of a Continuous Gaussian Process to a General Boundary," Journal of Applied Probability, Vol. 22, No. 1, 1985, pp. 99-122. doi: $10.2307 / 3213751$

[8] P. Salminen, "On the First Hitting Time and the Last Exit Time for a Brownian Motion to/from a Moving Boundary," Advances in Applied Probability, Vol. 20, No. 2, 1988, pp. 411-426. doi:10.2307/1427397

[9] A. Martin-Löf, "The Final Size of a Nearly Critical Epidemic and the First Passage Time of a Wiener Process to a Parabolic Barrier," Journal of Applied Probability, Vol. 35, No. 3, 1998, pp. 671-682. doi: $10.1239 / \mathrm{jap} / 1032265215$

[10] L. Breiman, "Probability," SIAM, Philadelphia, 1992. doi:10.1137/1.9781611971286

[11] J. Kevorkian, "Partial Differential Equations: Analytical Solution Techniques," Wadsworth, Belmont, 1990.

[12] F. John, "Partial Differential Equations," 4th Edition, Springer-Verlag, New York, 1982.

[13] S. M. Ross, "Stochastic Processes," John Wiley \& Sons, New York, 1983. 